Rev. Elev. Méd. vét. Pays trop., 1974, 27 (3) : 271-274

\title{
Stabilisation du lait par addition de Bêta-propiolactone
}

\author{
par J. BLANCOU (*)
}

\begin{abstract}
RESUME
L'addition de 0,5 p. 1000 Bêta-propiolactone au lait permet de le conserver durant au moins 48 heures de plus qu'un lait témoin non traité. Ce produit paraît être plus efficace que les antiseptiques actuellement utilisables dans le même but.
\end{abstract}

\section{INTRODUCTION}

Les techniques de conservation du lait autres que l'emploi du froid, de la chaleur ou de la dessiccation, sont généralement peu utilisées. L'addition d'antiseptique, du fait de son interdiction systématique par certaines législations, a fait l'objet de peu de recherches et d'applications directes. Les procédés pratiquement utilisables sont rares: R. VEISSEYRE, dans son ouvrage «Techniques laitières modernes 》 (8), ne cite que les méthodes à l'eau oxygénée et à la chloropicrine.

Néanmoins ces techniques gagneraient à être étudiées et développées en pays tropicaux où, comme le souligne H. LUCCK, l'altération du lait est rapide et la technologie du froid insuffisamment développée ou trop coûteuse (4).

Leur but n'est pas une stérilisation totale du lait, mais une simple * stabilisation *, c'est-àdire une prolongation de ses délais de conservation après la traite.

En effet, la stérilisation totale du lait par les antiseptiques, aisément réalisable au plan technique, altère le plus souvent l'équilibre physicochimique du produit ou ses propriétés biologiques. Elle confère surtout un sentiment de fausse sécurité au producteur, aux dépens des

(*) Laboratoire Central de l'Elevage, Service de Bactériologie, B.P. 4, Tananarive, République Malgache. mesures élémentaires d'hygiène qu'il lui est indispensable de rechercher par ailleurs.

La puissante valeur bactéricide de la bêtapropiolactone et sa stabilité chimique permettent de doser avec exactitude ses concentrations optimales, ce qui n'est pas possible avec l'eau oxygénée par exemple (7).

Nous décrirons donc, dans la présente note, les résultats obtenus par l'addition de Bêtapropiolactone au lait, en précisant ses avantages, ses inconvénients et ses limites.

\section{MATERIEL ET METHODE}

\section{MATERIEL}

\section{Lait}

Nous avons employé du lait de vache, obtenu par traite manuelle ou mécanique, et pollué à divers degrés (cf. tableaux annexes).

Le lait est traité aussitôt après sa récolte, sans subir aucun traitement intermédiaire par le froid ou la chaleur.

\section{Bêta-propiolactone}

Nous avons employé de la Bêta-propiolactone * purum \& de formule (*).

$$
\mathrm{O}-\mathrm{CH} 2-\mathrm{CH} 2-\mathrm{CO}
$$

(*) Etablissement FLUKA. 
- Poids moléculaire : 72,06.

- Point d'ébullition : $47^{\circ}-5^{\circ}$.

- Densité à $20^{\circ}: 1,14$.

Ce produit a la propriété de s'hydrolyser rapidement, au contact des solutions aqueuses, en dérivés de l'acide propionique et hydracrilique. L'hydrolyse est totale en 20 minutes à $37^{\circ}$ et $\mathrm{pH} 7$ (3), à la concentration de 0,6 p. 1000 .

Cette propriété est largement mise à profit, en microbiologie, pour neutraliser les cultures virulentes (6) qui perdent alors leur pouvoir dysgénésique pour les bactéries dès que l'hydrolyse est achevée.

\section{METHODES}

\section{Expériences préliminaires}

Sur des ballons de $250 \mathrm{ml}$ de lait, contenant en moyenne $10^{6,4}$ bactéries par $\mathrm{ml}$, nous avons fait agir différentes quantités de Bêta-propiolactone, aux dilutions finales suivantes:

$0,125-0,25-0,5-1-2$ et 5 pour mille ml de lait.

Des analyses bactériologiques successives, consécutives à l'addition de ces différentes quantités, nous ont montré que la stérilisation du lait était complète au-delà d'une concentration finale de 2 p. 1000 . L'acidité ionique du lait ainsi traité s'abaisse alors à des valeurs inférieures à $\mathrm{pH} 5$, et ses qualités organoleptiques sont modifiées.

\section{Expériences définitives}

Deux types d'essais ont été réalisés :

1. Essai sur des échantillons de $250 \mathrm{ml}$, en ballons stériles et bouchés au coton.

2. Essai sur des échantillons commerciaux de $2500 \mathrm{ml}$, en bidons simplement lavés et bouchés à vis.

Dans les deux types d'essais les échantillons ont été soumis, avant et après addition de $0,5 \mathrm{ml}$ de Bêta-propiolactone $\left(^{*}\right)$, à une analyse

(*) Cette concentration, qui n'entraîne pas une stérilisation totale du lait, a été retenue pour des raisons pratiques. Elle permet, en effet, une stabilisation suffisante du lait ( 48 heures) tout en restant d'un prix de revient inférieur à celui d'autres antiseptiques. bactériologique et à une évaluation de leurs caractères physico-chimiques et organoleptiques. L'échantillon étant conservé à température ambiante $\left(18^{\circ}\right.$ à $\left.24^{\circ}\right)$, les analyses sont faites après $12-24-48$ et 72 heures.

\section{A. Analyse bactériologique}

La microflore bactérienne est évaluée de deux façons :

1. Numérations de la flore aéro-anaérobie mésophile totale, par ensemencement des différentes dilutions de lait $\left(10^{-1}\right.$ à $\left.10^{-12}\right)$ en gélose nutritive standard incubée à $32^{\circ}$ durant 72 heures $(2,5)$.

2. Numérations de certaines bactéries aérobies ou anaérobies spécifiques: Coliformes et Clostridium sulfito-réducteurs. Ces numérations sont faites par ensemencement des dilutions précédentes sur milieu au Désoxycholate et sur milieu de Wilson Blair.

\section{B. Caractères physico-chimiques et organoleptiques}

- L'odeur, la couleur, la viscosité et la saveur du lait sont évaluées par comparaison avec celles d'un échantillon témoin.

- L'acidité ionique du lait est évaluée au $\mathrm{pH}$ mètre électrique.

\section{RESULTATS}

Le résultat de vingt-cinq analyses, faites soit en ballons de $250 \mathrm{ml}$ soit en bidons de $2500 \mathrm{ml}$, a été résumé dans les tableaux I et II.

Ces tableaux font état des résultats de l'analyse bactériologique et des mesures du $\mathrm{pH}$. Lorsque les caractères physico-chimiques et organoleptiques de l'échantillon témoin et de l'échantillon additionné du Bêta-propiolactone diffèrent notablement, cette différence est également indiquée $\left(^{*}\right)$.

Par rapport à la normale, cette différence est essentiellement une question d'acidité (perceptible au goût), d'odeur (fermentation lactique) et d'aspect physique (formation de grumeaux).

\section{DISCUSSION}

L'addition du Bêta-propiolactone au lait permet de «stabiliser» ce produit durant 
TABLEAU $N^{*}$ I

Egsais en ballons stériles ( $250 \mathrm{~m} 1$ ) hermétiques

\begin{tabular}{|c|c|c|}
\hline \multicolumn{3}{|c|}{ pH et wicro-flore aéro-anaérobie résophile totale par ml de lait } \\
\hline Temps Lait & Lait têmoin & Lait additionné de B.P.L \\
\hline Avant traitement & $\mathrm{pH}: 5,9$ & $\mathrm{pH}: 5,5$ \\
\hline 24 heures après & $\mathrm{pH}: 5,6$ & $\mathrm{pH}: 5,7$ \\
\hline 48 heures après & $10^{8,2 r}$ & $\mathrm{pH}: 5,5$ \\
\hline 72 heures après & $\mathrm{pH}: 4,8 \quad 10^{9} \mathrm{r}$ & $\mathrm{pH}: 5,5$ \\
\hline Après 72 heures & $\mathrm{pH}: 4,6$ & $\mathrm{pH}: 5,2$ \\
\hline \multicolumn{3}{|c|}{ Micro-flore spẹcifique par ml de lait } \\
\hline Avant traitement & $\begin{array}{ll}\text { Coliformes : } & 10^{4,8} \\
\text { Streptocoques } & 10^{4,1} \\
\text { Clootridium sulfito- } & \\
\text { rêducteurs } & 10^{1,2}\end{array}$ & $\begin{array}{l}\text { Coliformes : } \\
\text { Clostridium sulfito- } \\
\text { rêducteurs }\end{array}$ \\
\hline 24 heures après & $\begin{array}{ll}\text { Coliformes : } & 10^{6} \\
\text { Clostridium sulfito- } & \\
\text { réducteurs } & 10^{1,2}\end{array}$ & $\begin{array}{ll}\text { Coliformes : } & 10^{2,1} \\
\text { Clostridium sulfito- } & \\
\text { rêducteurs } & \text { absence }\end{array}$ \\
\hline 48 heures après & $\begin{array}{l}\text { Coliformes : } 10^{6,1} \\
\text { Clastridiwn sulfito- } \\
\text { rêducteurs }\end{array}$ & $\begin{array}{ll}\text { Coliformes : } & 10^{3,5} \\
\text { Clostridium sulfito- } & \\
\text { réducteurs } & \text { absence }\end{array}$ \\
\hline
\end{tabular}

* Lait coagulê en masse, odeur, viscositê et saveur anormale et inacceptable.

Rectification : supprimer «Streptocoques $10^{4,1}$ ».

TABLEAU $N^{*}$ II

Essais en bidons lavés ( $2500 \mathrm{ml}$ ) non hermétiques

\begin{tabular}{|c|c|c|}
\hline \multicolumn{3}{|c|}{ pH et micro-flore aéro-anaérobies mésophile par ml de lait } \\
\hline Temps & Lait têmoin & Lait additionné de B.P.L \\
\hline Avant traitement & $\mathrm{pH}: 5,8$ & $\mathrm{pH}: 5,6$ \\
\hline 24 heures après & $\mathrm{pH}: 5,7$ & $\mathrm{pH}: 5,9$ \\
\hline 48 heures après & $\mathrm{pH}: 5,3$ & $\mathrm{pH}: 5,6$ \\
\hline 72 heures après & $10^{9,5 \mathrm{H}}$ & $\mathrm{pH}: 5,5$ \\
\hline Après 72 heures & $\mathrm{pH}: 4,5$ & $\mathrm{pH}: 5,4$ \\
\hline \multicolumn{3}{|c|}{ Micro-flore spécifique par ml de lait } \\
\hline Avant traitement & $\begin{array}{l}\text { Colıformes: } 10^{5} \\
\begin{array}{l}\text { Clostridium sulfito- } \\
\text { rêducteurs }\end{array} 1^{1,5}\end{array}$ & $\begin{array}{l}\text { Coliformes : } 10^{5} \\
\text { Clostridium oulfito- } \\
\text { rêducteurs }\end{array}$ \\
\hline 24 heures aprè & $\begin{array}{l}\text { Coliformes : } 10^{6,1} \\
\begin{array}{l}\text { Clostridium sulfito- } \\
\text { réducteurs }\end{array}\end{array}$ & $\begin{array}{l}\text { Coliformes : } 10^{2,4} \\
\text { Clostridium sulfito- } \\
\text { rêducteurs }\end{array}$ \\
\hline 48 heures après & $\begin{array}{ll}\text { Coliformes : } & 10^{6,7} \\
\text { Clostmidium sulfito- } & \\
\text { réducteurs } & 10^{1,8}\end{array}$ & $\begin{array}{ll}\text { Coliformes : } & 10^{3,3} \\
\text { Clostridium sulfito- } \\
\text { rêducteurs }\end{array}$ \\
\hline
\end{tabular}

3 Lait coaguié en masse.

Rectification: $3^{\mathrm{e}}$ colonne, $3^{\mathrm{e}}$ ligne, lire $10^{2,1}$ au lieu de $10^{2,1 *}$. 
48 heures au moins, même en bidons non hermétiquement clos. Cet antiseptique paraît plus efficace, car plus puissant, que les antiseptiques habituels, à prix égal.

Un seul problème se pose : celui de la toxicité éventuelle de la Bêta-propiolactone ou de ses produits d'hydrolyse.

Il convient donc de préciser que la Bêtapropiolactone est totalement hydrolysée en milieu aqueux, ainsi que des titrages de produit résiduel peuvent le démontrer (1). Ce point est important, les propriétés cancérigènes de la Bêta-propiolactone étant parfois évoquées.

La toxicité des produits d'hydrolyse (dérivés de l'acide hydracrilique et propionique) n'a pu être démontrée jusqu'ici.
L'injection parentérale de vaccins traités à la Bêta-propiolactone n'a jamais entraîné d'effets toxiques, immédiats ou retardés, chez les sujets vaccinés $(6,3)$. Nous avons nous-mêmes recontrôlé cette absence de toxicité en abreuvant quotidiennement, durant 21 jours, des moutons et des souris avec des solutions à 10 p. $100 \mathrm{de}$ Bêta-propiolactone. Et nous avons nous-mêmes ingéré à plusieurs reprises des quantités importantes de lait «stabilisé » pour 1 p. 100 de Bêta-propiolactone sans aucun trouble digestif.

Néanmoins, le procédé de stabilisation pourrait être réservé, par mesure de précaution, à des laits destinés à l'alimentation animale. Dans cette seule perspective, l'intérêt du procédé recevrait nombre d'applications pratiques importantes.

\title{
SUMMARY
}

Milk stabilization by addition of beta-propiolactone

\begin{abstract}
The addition of 0,5 p. 1000 beta-propiolactone to milk allows to preserve it during at least 48 hours more than a no treated reference milk. This product seems to be more effective than antiseptics now available with this object.
\end{abstract}

\section{RESUMEN}

\section{Esterilización de la leche por añadido de beta-propiolactone}

El añadido de 0,5 p. 1000 beta-propiolactone en la leche permite conservarla durante a lo menos 48 horas más que una leche testigo no tratada. Este producto parece ser más eficaz que los asépticos actualmente utilizables para el mismo propósito.

\section{BIBLIOGRAPHIE}

1. FAYET (M. T.), PETERMANN (H.G.), FONTAINE (J.), TERRE (J.) et ROUMIANTZEFF (M.). Utilisations de la Bêta-propiolactone comme agent d'inactivation pour la préparation des vaccins contre la fièvre aphteuse. Ann. Inst. Pasteur, 1967,112 (1) : 65-76.

2. FOSTER (E.M.), NELSON (F.E.), SPECK (M.L.), DOETSCH (R. N.) et OLSON (J. C.). Dairy microbiology. Englewood Clifs, N.J. (U.S.A.), Prentice Hall, 1957.

3. GUILLOTEAU (B.). Vaccins anti-rabiques formolés et lactonés d'usage vétérinaire. Thèse Méd. vét. Alfort, 1963, no 75 .

4. O.M.S. Hygiène du lait. Genève, F.A.O./O.M.S., 1966.
5. PETRANSXIENNE (D.) et LAPIED (L.). La quantité bactériologique du lait, F.H.P.L., Paris, Goujon, 1962.

6. RAYNAUD (M.), BLASS (J.) et TURPIN (A.). Mécanisme de la détoxification des toxines par le formol. Etude des deux nouveaux dérivés atoxiques antigéniques: $2-4$ démitrofluorobenzène toxoïde et Bêta-propiolactone toxoïde. C.R. Acad. Sci., 1967, 24 : 862-863.

7. RIBOT (J. J.) et SERRES (H.). L'eau oxygénée, agent bactéricide et bactériostatique des laits malgaches. Journées médicales Tananarive. In: Rapport annuel Laboratoire Central Elevage 1968. Archives I.E.M.V.T. et C.N.R.Z.M.V., Tananarive.

8. VEISSEYRE (R.). Techniques laitières modernes. Paris, La Maison rustique, 1957. 